\title{
The low density and magnetization of a massive galaxy halo exposed by a fast radio burst
}

\author{
J. Xavier Prochaska ${ }^{1,2 *}$, Jean-Pierre Macquart ${ }^{3}$, Matthew McQuinn ${ }^{4}$, Sunil Simha', Ryan M. Shannon ${ }^{5}$, Cherie \\ K. Day ${ }^{5,6}$, Lachlan Marnoch ${ }^{6,7}$, Stuart Ryder ${ }^{7}$, Adam Deller ${ }^{5}$, Keith W. Bannister ${ }^{6}$, Shivani Bhandari ${ }^{6}$, Rongmon \\ Bordoloi $^{8}$, John Bunton ${ }^{6}$, Hyerin Cho ${ }^{9}$, Chris Flynn ${ }^{5}$, Elizabeth K. Mahony ${ }^{6}$, Chris Phillips ${ }^{6}$, Hao Qiu ${ }^{10}$, Nicolas \\ Tejos $^{11}$
}

${ }^{1}$ University of California Observatories-Lick Observatory, University of California, 1156 High Street, Santa Cruz, CA 95064, USA. ${ }^{2}$ Kavli Institute for the Physics and Mathematics of the Universe, 5-1-5 Kashiwanoha, Kashiwa, 277-8583, Japan. ${ }^{3}$ International Centre for Radio Astronomy Research, Curtin University, Bentley, WA 6102, Australia. ${ }^{4}$ Astronomy Department, University of Washington, Seattle, WA 98195, USA. ${ }^{5}$ Centre for Astrophysics and Supercomputing, Swinburne University of Technology, Hawthorn, VIC 3122, Australia. ${ }^{6}$ Commonwealth Science and Industrial Research Organisation, Australia Telescope National Facility, P.0. Box 76, Epping, NSW 1710 Australia. ${ }^{7}$ Department of Physics and Astronomy, Macquarie University, NSW 2109, Australia. ${ }^{8}$ North Carolina State University, Department of Physics, Raleigh, NC $27695-$ 8202, USA. 'School of Physics and Chemistry, Gwangju Institute of Science and Technology, Gwangju, 61005, Korea. ${ }^{10}$ Sydney Institute for Astronomy, School of Physics, University of Sydney, Sydney, NSW 2006, Australia. "'Instituto de Física, Pontificia Universidad Católica de Valparaíso, Casilla 4059, Valparaíso, Chile.

*Corresponding author. Email: xavier@ucolick.org

Present-day galaxies are surrounded by cool and enriched halo gas extending for hundreds of kiloparsecs. This halo gas is thought to be the dominant reservoir of material available to fuel future star formation, but direct constraints on its mass and physical properties have been difficult to obtain. We report the detection of a fast radio burst (FRB 181112), localized with arcsecond precision, which passes through the halo of a foreground galaxy. Analysis of the burst shows the halo gas has low net magnetization and turbulence. Our results imply predominantly diffuse gas in massive galactic halos, even those hosting active supermassive black holes, contrary to some previous results.

The low-density gas located in the outskirts of galaxies influences the process of galaxy formation, especially gas accretion and feedback (1). Absorption-line spectroscopy can detect this nearly invisible medium. Surveys demonstrate a very high incidence of cool gas (with temperature $T \sim 10^{4} \mathrm{~K}$ ), detected through hydrogen Lyman series and continuum absorption, surrounding galaxies with masses similar to our Milky Way (e.g., $(1,2))$. Properties of this gas depend on galaxy mass but are otherwise insensitive to the galaxy's internal properties (1,3-5). Estimates for the total mass of the cool gas match or exceed the baryonic mass of the galaxy $(4,6)$. Theoretical treatments of halo gas around present-day galaxies disagree on the proportion of total mass retained in the halo during galaxy formation, with estimates ranging from several tens of percent up to all of the baryons predicted to accrete into the halo (e.g., $(7,8))$. This uncertainty stems from observational insensitivity to the hot $\left(T \gtrsim 10^{6} \mathrm{~K}\right)$ gas which pervades galaxy halos (and within which the cold gas is embedded), and from systematic uncertainties in estimating its mass $(1,6)$. Constraints on the density and temperature of the halo gas are sufficiently limited to allow qualitatively different descriptions of its ionization and distribution $(9,10)$. The origin of the cool gas and its composition are challenging to explain theoretically; some models require cosmic rays and magnetic fields to transport material from the central galaxy to sustain the cool medium (e.g., (11)).
At Coordinated Universal Time 17:31:15.48365 on 2018 Nov 12 the Commensal Real-time ASKAP Fast Transients (CRAFT) survey on the Australian Square Kilometer Array Pathfinder (ASKAP) detected a fast radio burst (FRB 181112) from the 12 antennas observing at the time. The burst arrival time swept across the observing band $(\approx 1.129-1.465 \mathrm{GHz}$, Fig. 1A) due to propagation of the burst through a foreground plasma. The burst sweep yields an estimate of the FRB dispersion measure $\mathrm{DM}_{\mathrm{FRB}}=589.27 \pm 0.03 \mathrm{pc} \mathrm{cm}^{-3}$ which is the integrated density of electrons $n_{e}$ at distance $r$ from Earth scaled by $(1+z)^{-1}$ with $z$ the redshift: $\mathrm{DM}_{\mathrm{FRB}} \equiv \int n_{e} /(1+z) d r$. The real-time detection triggered full download of the voltage data; these precisely localized the burst to a sky position $21 \mathrm{~h} 49 \mathrm{~m} 23.630 \mathrm{~s},-52 \mathrm{~d} 58 \mathrm{~m} 15.39 \mathrm{~s}$ (right ascension, declination, J2000 equinox) with a statistical (systematic) error ellipse oriented at 120 deg $\mathrm{E}$ of $\mathrm{N}$ on the sky with major axis $a=$ $0.555^{\prime \prime}\left(3.2^{\prime \prime}\right)$ and minor axis $b=0.153^{\prime \prime}\left(0.8^{\prime \prime}\right)(12)$.

Figure 1B shows a $g$-band image centered on FRB 181112 obtained with the FOcal Reducer/low dispersion Spectrograph 2 (FORS2) instrument on the Very Large Telescope (VLT). It shows the presence of a galaxy coincident with FRB 181112, previously cataloged by the Dark Energy Survey (DES; (13)) as DES J214923.66-525815.28. The DES and FORS2 data also show a luminous galaxy $\approx 5^{\prime \prime}$ to the North of the FRB event (DES J214923.89-525810.43). We used follow-up 
spectroscopy with the FORS2 instrument to measure the redshift (12) of the former galaxy as $z=0.47550$, and the latter galaxy as $z=0.3674$, i.e., in the foreground. We associate FRB 181112 with DES J214923.66-525815.28 (12). Compared to the other three known host galaxies of FRBs, the host galaxy of FRB 181112 has an intermediate stellar mass of $M_{\star} \approx 10^{9.4}$ solar masses $\left(M_{\odot}\right)$ (fig. S3) (12). It has colors matching starforming galaxies at $z \sim 0.4$, has an estimated star formation rate of $0.6 M_{\odot} \mathrm{yr}^{-1}$, and shows no signatures of an active galactic nucleus (AGN) (12).

The FRB sightline passes at an impact parameter $R_{\perp}=29 \mathrm{kpc}$ from DES J214923.89-525810.43 (hereafter referred to as FG-181112) allowing us to probe the halo of this foreground galaxy. We analyzed the DES, FORS2, and complementary longer-wavelength Wide-field Infrared Survey Explorer (WISE) data to determine FG-181112's physical properties (12), We derive a high stellar mass $\log _{10} M_{\star} / M_{\odot}=10.69_{-0.46}^{+0.22}$, detect nebular emission lines indicative of an AGN and classify it as a Seyfert galaxy, and infer an old (age $>1.4 \mathrm{Gyr}$ ) quiescent stellar population (Table 1 and table S5). Surveys of the halo gas surrounding galaxies of similar mass, with or without AGN activity (14), almost ubiquitously reveal strong absorption by $\operatorname{cool}\left(T \sim 10^{4} \mathrm{~K}\right)$ gas for sightlines $R_{\perp} \leq 100 \mathrm{kpc}$. Generally, the inferred total column densities of ionized gas exceed $10^{20} \mathrm{~cm}^{-2}(4,6)$, and transitions of heavy elements indicate a turbulent velocity field (15) suggesting that a fraction of the gas has a relatively high density $\left(n_{\mathrm{H}} \sim 1 \mathrm{~cm}^{-3} ;(16)\right)$. Such a foreground medium should impact the FRB signal.

The column of gas close to this massive galaxy, however, does not dominate $\mathrm{DM}_{\mathrm{FRB}}$. It contributes only $\mathrm{DM}_{\mathrm{FG}} \sim 50-$ $120 \mathrm{pc} \mathrm{cm}^{-3}$, depending on assumptions for the density profile and total mass of the halo gas (12). The measured $\mathrm{DM}_{\mathrm{FRB}}$ is consistent with models that include cosmic gas, our Galaxy, and the host (fig. S9) $(17,18)$. The sightline to FRB 181112 also intersects the edge of the Fermi Bubbles (12), a complex of hot gas encompassing the Galactic Center. The expected DM contribution from gas in these bubbles is small (12), but their entrained magnetic field may contribute to the FRB rotation measure.

The rotation measure $\mathrm{RM}$ is the density-weighted integral of the magnetic field parallel to the FRB sightline. The voltages recorded from the ASKAP antennas measure the electric field at the antenna locations in two orthogonal directions on the plane of the sky, enabling the linear polarization fraction of the burst radiation (and its position angle) to be measured as a function of frequency. Averaged over its duration we find the burst to be approximately $90 \%$ linearly polarized and $10 \%$ circularly polarized (12). This can be used to estimate the burst $\mathrm{RM}$, as $\mathrm{PA}_{\mathrm{obs}}=\mathrm{PA}_{\text {int }}+(c / v)^{2} \mathrm{RM}$, where $v$ is the frequency and $\mathrm{PA}_{\mathrm{obs}}$ and $\mathrm{PA}_{\text {int }}$ are the observed and intrinsic polarization angles, respectively. Figure 2 depicts the frequency sweep of the polarization angle; the apparent $v^{-2}$ frequency dependence is the RM signature. We fitted an RM model to the sweep, yielding $\mathrm{RM}=10.9 \pm 0.9 \mathrm{rad} \mathrm{\textrm {m } ^ { - 2 }}$. This is a low $\mathrm{RM}$ value, consistent (within the uncertainty) with the estimated RM due to our Galaxy toward FRB 181112 (12). Adopting an upper limit of $\mathrm{RM}<11 \mathrm{rad} \mathrm{m}^{-2}$, we calculate an upper limit for the maximum parallel magnetic field $B_{\|}^{\max }$ in the halo of FG-181112: $B_{\|}^{\max }<0.8 \mu \mathrm{G}\left(n_{e} / 10^{-3} \mathrm{~cm}^{-3}\right)^{-1}(\Delta L / 30 \mathrm{kpc})^{-1}$, in the limit of a perfectly ordered magnetic field with $\Delta L$ a characteristic length-scale through the halo. We have adopted fiducial values for $n_{e}$ and $L$ that may characterize the halo of FG181112 (similar to those adopted for the $\mathrm{DM}_{\mathrm{FG}}$ estimation). Field reversals would lead us to underestimate $B_{\|}^{\max }$. Never-

theless, this low value for $B_{\|}^{\max }$ implies that either the magnetic field in the halo is low compared to the interstellar medium, or that it is largely disordered.

These constraints have implications for the circumgalactic gas. The magnetic field value in equipartition with the thermal energy of the virialized halo gas is $B_{\text {eq }} \equiv \sqrt{8 \pi n_{e} k_{b} T}=2 \mu \mathrm{G}\left(n_{e} / 10^{-3} \mathrm{~cm}^{-3}\right)^{1 / 2}\left(T / 10^{6} \mathrm{~K}\right)^{1 / 2}$ with $k_{b}$ the Boltzmann constant. Our $B_{\|}^{\max }$ limit is similar to $B_{\text {eq }}$ for physically motivated $n_{e}, \Delta L$ and $T$, constraining the magnetic field to be near or below equipartition if the total field is similar to the net parallel field. Magnetic fields around the equipartition value enhance the rate of condensation of the hot circumgalactic gas into cooler clouds (11) as well as the survival of cool accreting gas (19). Near equipartition field strengths are generated in some models in which cosmic ray pressure transports cool gas and metals to large distances from galaxies $(20,21)$. Our limit on $B_{\|}^{\max }$ is below the mean estimate for sightlines that show strong gas absorption (22) despite our sightline likely intersecting gas with similarly strong absorption in FG-181112 (e.g., (3)).

The halo gas of FG-181112 broadens the width of the pulse at any given frequency. This temporal broadening $\tau_{\text {scatt }}$ arises from density fluctuations within the medium which impose small differences in light-travel time for rays propagating through the gas $(17,23)$. This scattering is geometrical and its effects are maximal for a scattering "screen" located at onehalf the distance to the FRB. We determine an upper limit of $\tau_{\text {scatt }}<40 \mu$ s due to scattering, constraining both the turbulent properties of the halo gas and its density. A pulse with 150 times higher width ( $3 \mathrm{~ms}$ ) would have still been detected, i.e., the very narrow width of FRB 181112 is not the result of observational bias. Figure $2 \mathrm{~B}$ shows that the temporal profile of FRB 181112 consists of two pulses separated by approximately 
$800 \mu \mathrm{s}$. The broadening limit is derived by modeling each component as a symmetric intrinsic pulse convolved with the one-sided exponential decay expected due to scattering (see (12)). Temporal smearing due to inhomogeneities in the plasma distribution along the line of sight would otherwise broaden the pulse to a frequency-dependent duration $\tau_{\text {scatt }}(v)$ $=\tau_{0}(v / 1 \mathrm{GHz})^{\gamma}$, where the index $\gamma$ is typically $\approx-4(12)$.

The observed $\tau_{\text {scatt }}$ constrains the integral of the square of the density along the sightline, $\int d x \delta n_{e}(x)^{2}$, which we relate to the electron column density with the parameterization $\left\langle n_{e}\right\rangle \Delta L^{1 / 2}=\alpha^{-1}\left(\int d x \delta n_{e}^{2}\right)^{1 / 2}$, which takes the halo of FG-181112 to have characteristic length $\Delta L$ with an average density of $\left\langle n_{e}\right\rangle$. Thus, the parameter $\alpha$ encapsulates the root-meansquare amplitude of density fluctuations and the volume filling fraction of the turbulence, $f_{V}$. The limit on the in situ density assuming a Kolmogorov spectrum of turbulence (12) is

$$
\left\langle n_{e}\right\rangle<2 \times 10^{-3} \alpha^{-1}\left(\frac{\Delta L}{50 \mathrm{kpc}}\right)^{-1 / 2}\left(\frac{L_{0}}{1 \mathrm{kpc}}\right)\left(\frac{\tau_{\text {scatt }}}{40 \mu \mathrm{s}}\right)^{5 / 12} \mathrm{~cm}^{-3}
$$

where $\Delta L \sim 50 \mathrm{kpc}$ approximates the path length through the foreground halo and $L_{0}$ is the outer scale of turbulence. As the turbulence is likely to be produced by galactic winds and inflows, we expect it to be driven at scales less than the impact parameter $(\sim 30 \mathrm{kpc})$ and consider $L_{0}=1 \mathrm{kpc}$ a reasonable value.

We now examine two standard models for halo gas in which the medium is comprised of either hot $\left(T \sim 10^{6} \mathrm{~K}\right)$ virialized gas or cool gas pressure-confined by the hot gas. In the case of hot virialized gas, our constraint on $\left\langle n_{e}\right\rangle$ suggests densities lower than those expected of $\sim 10^{-3} \mathrm{~cm}^{-3}$ gas with kiloparsec driving scales (see fig. S12). Because we expect the volume filling factor of this gas to be near unity, the upper limit on the density can only be ameliorated if the gas is much less turbulent (i.e., $\alpha \ll 1$ ) relative to galactic astrophysical plasmas, especially the interstellar medium of our Galaxy, where $\alpha \sim 7(12,24)$.

For turbulent, cool $10^{4} \mathrm{~K}$ clouds embedded in a hot medium, the constraints are stronger. Assuming pressure equilibrium with characteristic values for the hot gas $n_{e}=10^{-3}$ $\mathrm{cm}^{-3}$ and $T=2 \times 10^{6} \mathrm{~K}$, application of Eq. 1 with $L_{0}=1 \mathrm{kpc}$ and $\Delta L=50 \mathrm{kpc}$ yields $\alpha<0.01$. Because $\alpha \propto f_{V}^{1 / 2}$, we require a filling factor of cool clouds of $f_{V}<10^{-4}$ if the clouds are fully turbulent. Even lower values are required to satisfy this condition if the driving for turbulence within cool clouds is instead at parsec scales, which may be physically motivated (25).

These limits on the halo gas density derived from the scattering analysis contradict prior inferences that cool halo gas has a volume filling fraction of $f_{V} \sim 10^{-3}-10^{-2}(6,26,27)$. The total neutral hydrogen column density offers the most direct comparison to our result: photoionization equilibrium constrains the same combination of parameters as scattering, implying $\left(n_{e} / 0.1 \mathrm{~cm}^{-3}\right)\left(f_{V} / 10^{-3}\right)^{1 / 2}(\Delta L / 50 \mathrm{kpc})^{1 / 2} \sim 1$ if we take a typical neutral hydrogen column density of $10^{18} \mathrm{~cm}^{-2}$ at 30 kpc measured for halos with similar masses as FG-181112 (4). Reconciling these values with the scattering from FG-181112 either implies that the cool clouds are less turbulent than assumed or that our sight line has less cool gas than is typical. The foreground galaxy is classified as a Seyfert, with an embedded accreting supermassive black hole in a central AGN that could lead to a more evacuated halo (28), although it has been argued that such activity may lead to more cool gas (29). Even if the clouds are not turbulent and instead we consider the refractive bending of light through a network of parsecscale clouds (25), we rule out a population of $0.1 \mathrm{pc}$ clouds or smaller with $f_{V} \sim 10^{-3}(12)$.

FRBs experience a number of propagation effects which render them sensitive probes of the density, magnetic field and turbulence of the otherwise elusive gas that pervades galaxy halos. The constraints derived from FRB 181112 for the halo of a massive galaxy are summarized in Fig. 3. The $n_{e}, B$ parameter space ruled out by our observations conflicts with several previous inferences for halo gas $(22,26,27)$. Our observations indicate a density of hot gas that is lower than in many models and also a column of cool gas that is smaller than commonly inferred.

Our results demonstrate that FRBs can be used to elucidate the physical properties of diffuse gas in the halos of galaxies. The multiple pulses observed in FRB 181112 could be due to multipath propagation through the gas. That would be a natural consequence of a medium comprising very low filling-factor cool clouds embedded in hot virialized halo gas, with the pulse multiplicity signifying the number of clouds intersected, and their arrival times yielding their offsets from the direct burst sight line.

\section{REFERENCES AND NOTES}

1. J. Tumlinson, M. S. Peeples, J. K. Werk, The Circumgalactic Medium. Annu. Rev. Astron. Astrophys. 55, 389-432 (2017). doi:10.1146/annurev-astro-091916055240

2. J. X. Prochaska, B. Weiner, H.-W. Chen, J. Mulchaey, K. Cooksey, Probing the intergalactic medium/galaxy connection. V. On the origin of $\mathrm{Ly} \alpha$ and $\mathrm{O} \mathrm{VI}$ absorption at $z<0.2$. Astrophys. J. 740, 91 (2011). doi:10.1088/0004$637 X / 740 / 2 / 91$

3. C. Thom, J. Tumlinson, J. K. Werk, J. Xavier Prochaska, B. D. Oppenheimer, M. S. Peeples, T. M. Tripp, N. S. Katz, J. M. O’Meara, A. B. Ford, R. Davé, K. R. Sembach, D. H. Weinberg, Not dead yet: Cool circumgalactic gas in the halos of early-type galaxies. Astrophys. J. 758, L41 (2012). doi:10.1088/2041-8205/758/2/L41

4. J. X. Prochaska, J. K. Werk, G. Worseck, T. M. Tripp, J. Tumlinson, J. N. Burchett, A. J. Fox, M. Fumagalli, N. Lehner, M. S. Peeples, N. Tejos, The COS-Halos Survey: Metallicities in the Low-redshift Circumgalactic Medium. Astrophys. J. 837, 169 (2017). doi:10.3847/1538-4357/aa6007

5. R. Bordoloi, J. X. Prochaska, J. Tumlinson, J. K. Werk, T. M. Tripp, J. N. Burchett, On the CGM Fundamental Plane: The Halo Mass Dependency of Circumgalactic $\mathrm{H}$ i. 
Astrophys. J. 864, 132 (2018). doi:10.3847/1538-4357/aad8ac

6. J. K. Werk, J. X. Prochaska, J. Tumlinson, M. S. Peeples, T. M. Tripp, A. J. Fox, N. Lehner, C. Thom, J. M. O'Meara, A. B. Ford, R. Bordoloi, N. Katz, N. Tejos, B. D. Oppenheimer, R. Davé, D. H. Weinberg, The COS-Halos survey: Physical conditions and baryonic mass in the low-redshift circumgalactic medium. Astrophys. J. 792, 8 (2014). doi:10.1088/0004-637X/792/1/8

7. Z. Hafen, C.-A. Faucher-Giguère, D. Anglés-Alcázar, J. Stern, D. Kereš, C. Hummels, C. Esmerian, S. Garrison-Kimmel, K. El-Badry, A. Wetzel, T. K. Chan, P. F. Hopkins, N. Murray, The origins of the circumgalactic medium in the FIRE simulations. Mon. Not. R. Astron. Soc. 488, 1248-1272 (2019). doi:10.1093/mnras/stz1773

8. A. Pillepich, V. Springel, D. Nelson, S. Genel, J. Naiman, R. Pakmor, L. Hernquist, P. Torrey, M. Vogelsberger, R. Weinberger, F. Marinacci, Simulating galaxy formation with the IllustrisTNG model. Mon. Not. R. Astron. Soc. 473, 4077-4106 (2018). doi:10.1093/mnras/stx2656

9. Y. Faerman, A. Sternberg, C. F. McKee, Massive warm/hot galaxy coronae as probed by uv/x-ray oxygen absorption and emission. I. Basic model. Astrophys. J. 835, 52 (2017). doi:10.3847/1538-4357/835/1/52

10. J. Stern, C.-A. Faucher-Giguère, J. F. Hennawi, Z. Hafen, S. D. Johnson, D. Fielding, Does Circumgalactic 0 vi Trace Low-pressure Gas Beyond the Accretion Shock? Clues from $\mathrm{H}$ i and Low-ion Absorption, Line Kinematics, and Dust Extinction. Astrophys. J. 865, 91 (2018). doi:10.3847/1538-4357/aac884

11. S. Ji, S. P. Oh, M. McCourt, The impact of magnetic fields on thermal instability. Mon. Not. R. Astron. Soc. 476, 852-867 (2018). doi:10.1093/mnras/sty293

12. Materials and methods are available as supplementary materials.

13. T. M. C. Abbott, F. B. Abdalla, S. Allam, A. Amara, J. Annis, J. Asorey, S. Avila, O. Ballester, M. Banerji, W. Barkhouse, L. Baruah, M. Baumer, K. Bechtol, M. R. Becker, A. Benoit-Lévy, G. M. Bernstein, E. Bertin, J. Blazek, S. Bocquet, D. Brooks, D. Brout, E. Buckley-Geer, D. L. Burke, V. Busti, R. Campisano, L. Cardiel-Sas, A. C. Rosell, M. C. Kind, J. Carretero, F. J. Castander, R. Cawthon, C. Chang, X. Chen, C. Conselice, G. Costa, M. Crocce, C. E. Cunha, C. B. D’Andrea, L. N. Costa, R. Das, G. Daues, T. M. Davis, C. Davis, J. D. Vicente, D. L. DePoy, J. DeRose, S. Desai, H. T. Diehl, J. P. Dietrich, S. Dodelson, P. Doel, A. Drlica-Wagner, T. F. Eifler, A. E. Elliott, A. E. Evrard, A. Farahi, A. F. Neto, E. Fernandez, D. A. Finley, B. Flaugher, R. J. Foley, P. Fosalba, D. N. Friedel, J. Frieman, J. García-Bellido, E. Gaztanaga, D. W. Gerdes, T. Giannantonio, M. S. S. Gill, K. Glazebrook, D. A. Goldstein, M. Gower, D. Gruen, R. A. Gruendl, J. Gschwend, R. R. Gupta, G. Gutierrez, S. Hamilton, W. G. Hartley, S. R. Hinton, J. M. Hislop, D. Hollowood, K. Honscheid, B. Hoyle, D. Huterer, B. Jain, D. J. James, T. Jeltema, M. W. G. Johnson, M. D. Johnson, T. Kacprzak, S. Kent, G. Khullar, M. Klein, A. Kovacs, A. M. G. Koziol, E. Krause, A. Kremin, R. Kron, K. Kuehn, S. Kuhlmann, N. Kuropatkin, O. Lahav, J. Lasker, T. S. Li, R. T. Li, A. R. Liddle, M. Lima, H. Lin, P. López-Reyes, N. MacCrann, M. A. G. Maia, J. D. Maloney, M. Manera, M. March, J. Marriner, J. L. Marshall, P. Martini, T. McClintock, T. McKay, R. G. McMahon, P. Melchior, F. Menanteau, C. J. Miller, R. Miquel, J. J. Mohr, E. Morganson, J. Mould, E. Neilsen, R. C. Nichol, F. Nogueira, B. Nord, P. Nugent, L. Nunes, R. L. C. Ogando, L. Old, A. B. Pace, A. Palmese, F. PazChinchón, H. V. Peiris, W. J. Percival, D. Petravick, A. A. Plazas, J. Poh, C. Pond, A. Porredon, A. Pujol, A. Refregier, K. Reil, P. M. Ricker, R. P. Rollins, A. K. Romer, A. Roodman, P. Rooney, A. J. Ross, E. S. Rykoff, M. Sako, M. L. Sanchez, E. Sanchez, B. Santiago, A. Saro, V. Scarpine, D. Scolnic, S. Serrano, I. Sevilla-Noarbe, E. Sheldon, N. Shipp, M. L. Silveira, M. Smith, R. C. Smith, J. A. Smith, M. SoaresSantos, F. Sobreira, J. Song, A. Stebbins, E. Suchyta, M. Sullivan, M. E. C. Swanson, G. Tarle, J. Thaler, D. Thomas, R. C. Thomas, M. A. Troxel, D. L. Tucker, V. Vikram, A. K. Vivas, A. R. Walker, R. H. Wechsler, J. Weller, W. Wester, R. C. Wolf, H. Wu, B. Yanny, A. Zenteno, Y. Zhang, J. Zuntz, S. Juneau, M. Fitzpatrick, R. Nikutta, D. Nidever, K. Olsen, A. Scott, The Dark Energy Survey: Data Release 1. Astrophys. J. 239, 18 (2018). doi:10.3847/1538-4365/aae9f0

14. T. A. M. Berg, S. L. Ellison, J. Tumlinson, B. D. Oppenheimer, R. Horton, R. Bordoloi, J. Schaye, The COS-AGN survey: Revealing the nature of circumgalactic gas around hosts of active galactic nuclei. Mon. Not. R. Astron. Soc. 478, 3890-3934 (2018). doi:10.1093/mnras/sty962

15. H.-W. Chen, V. Wild, J. L. Tinker, J.-R. Gauthier, J. E. Helsby, S. A. Shectman, I. B. Thompson, What determines the incidence and extent of Mg II absorbing gas around galaxies? Astrophys. J. 724, L176-L182 (2010). doi:10.1088/20418205/724/2/L176

16. T.-W. Lan, H. Mo, Exploring the physical properties of the cool circumgalactic medium with a semi-analytic model. Mon. Not. R. Astron. Soc. 486, 608-622 (2019). doi:10.1093/mnras/stz839

17. M. McQuinn, Locating the "missing" baryons with extragalactic dispersion measure estimates. Astrophys. J. 780, L33 (2014). doi:10.1088/20418205/780/2/L33

18. J. X. Prochaska, Y. Zheng, Probing Galactic haloes with fast radio bursts. Mon. Not. R. Astron. Soc. 485, 648-665 (2019). doi:10.1093/mnras/stz261

19. T. Berlok, C. Pfrommer, On the Kelvin-Helmholtz instability with smooth initial conditions - linear theory and simulations. Mon. Not. R. Astron. Soc. 485, $908-$ 923 (2019). doi:10.1093/mnras/stz379

20. R. Pakmor, C. Pfrommer, C. M. Simpson, V. Springel, Galactic winds driven by isotropic and anisotropic cosmic-ray diffusion in disk galaxies. Astrophys. J. 824, L30 (2016). doi:10.3847/2041-8205/824/2/L30

21. I. S. Butsky, T. R. Quinn, The Role of Cosmic-ray Transport in Shaping the Simulated Circumgalactic Medium. Astrophys. J. 868, 108 (2018). doi:10.3847/1538-4357/aaeac2

22. M. L. Bernet, F. Miniati, S. J. Lilly, P. P. Kronberg, M. Dessauges-Zavadsky, Strong magnetic fields in normal galaxies at high redshift. Nature 454, 302-304 (2008). doi:10.1038/nature07105 Medline

23. J.-P. Macquart, J. Y. Koay, Temporal smearing of transient radio sources by the intergalactic medium. Astrophys. J. 776, 125 (2013). doi:10.1088/0004$637 X / 776 / 2 / 125$

24. K. R. Anantharamaiah, R. Narayan, Radio Wave Scattering in the Interstellar Medium, J. M. Cordes, B. J. Rickett, D. C. Backer, Eds. (1988), vol. 174 of American Institute of Physics Conference Series, pp. 185-189.

25. M. McCourt, S. P. Oh, R. O'Leary, A.-M. Madigan, A characteristic scale for cold gas. Mon. Not. R. Astron. Soc. 473, 5407-5431 (2018) doi:10.1093/mnras/stx2687

26. S. Cantalupo, F. Arrigoni-Battaia, J. X. Prochaska, J. F. Hennawi, P. Madau, A cosmic web filament revealed in Lyman- $\alpha$ emission around a luminous highredshift quasar. Nature 506, 63-66 (2014). doi:10.1038/nature12898 Medline

27. T.-W. Lan, M. Fukugita, Mg ii Absorbers: Metallicity Evolution and Cloud Morphology. Astrophys. J. 850, 156 (2017). doi:10.3847/1538-4357/aa93eb

28. E. Choi, J. P. Ostriker, T. Naab, L. Oser, B. P. Moster, The impact of mechanical AGN feedback on the formation of massive early-type galaxies. Mon. Not. $R$. Astron. Soc. 449, 4105-4116 (2015). doi:10.1093/mnras/stv575

29. L. P. David, J. Lim, W. Forman, J. Vrtilek, F. Combes, P. Salome, A. Edge, S. Hamer, C. Jones, M. Sun, E. O'Sullivan, F. Gastaldello, S. Bardelli, P. Temi, H. Schmitt, Y. Ohyama, W. Mathews, F. Brighenti, S. Giacintucci, D.-V. Trung, Molecular gas in the x-ray bright group NGC 5044 as revealed by ALMA. Astrophys. J. 792, 94 (2014). doi:10.1088/0004-637X/792/2/94

30. R. Shannon, S. Bhandari, C. Day, E. Mahony, A. Deller, C. Phillips, FRB 181112 ATCA observations, V1, CSIRO Data Collection (2019); https://doi.org/10.25919/5d7e19c34743c.

31. J. X. Prochaska et al., FRBs/FRB: First DOI release of this repository, Version v1.0.0, Zenodo (2019); doi: http://doi.org/10.5281/zenodo.3403651.

32. P. A. R. Ade, N. Aghanim, M. Arnaud, M. Ashdown, J. Aumont, C. Baccigalupi, A. J. Banday, R. B. Barreiro, J. G. Bartlett, N. Bartolo, E. Battaner, R. Battye, K. Benabed, A. Benoît, A. Benoit-Lévy, J.-P. Bernard, M. Bersanelli, P. Bielewicz, J. J. Bock, A. Bonaldi, L. Bonavera, J. R. Bond, J. Borrill, F. R. Bouchet, F. Boulanger, M. Bucher, C. Burigana, R. C. Butler, E. Calabrese, J.-F. Cardoso, A. Catalano, A. Challinor, A. Chamballu, R.-R. Chary, H. C. Chiang, J. Chluba, P. R. Christensen, S. Church, D. L. Clements, S. Colombi, L. P. L. Colombo, C. Combet, A. Coulais, B. P. Crill, A. Curto, F. Cuttaia, L. Danese, R. D. Davies, R. J. Davis, P. de Bernardis, A. de Rosa, G. de Zotti, J. Delabrouille, F.-X. Désert, E. Di Valentino, C. Dickinson, J. M. Diego, K. Dolag, H. Dole, S. Donzelli, O. Doré, M. Douspis, A. Ducout, J. Dunkley, X. Dupac, G. Efstathiou, F. Elsner, T. A. EnBlin, H. K. Eriksen, M. Farhang, J. Fergusson, F. Finelli, O. Forni, M. Frailis, A. A. Fraisse, E. Franceschi, A. Frejsel, S. Galeotta, S. Galli, K. Ganga, C. Gauthier, M. Gerbino, T. Ghosh, M. Giard, Y. GiraudHéraud, E. Giusarma, E. Gjerløw, J. González-Nuevo, K. M. Górski, S. Gratton, A. Gregorio, A. Gruppuso, J. E. Gudmundsson, J. Hamann, F. K. Hansen, D. Hanson, D. L. Harrison, G. Helou, S. Henrot-Versillé, C. Hernández-Monteagudo, D. Herranz, S. R. Hildebrandt, E. Hivon, M. Hobson, W. A. Holmes, A. Hornstrup, W. Hovest, Z. Huang, K. M. Huffenberger, G. Hurier, A. H. Jaffe, T. R. Jaffe, W. C. Jones, M. Juvela, E. Keihänen, R. Keskitalo, T. S. Kisner, R. Kneissl, J. Knoche, L. 
Knox, M. Kunz, H. Kurki-Suonio, G. Lagache, A. Lähteenmäki, J.-M. Lamarre, A. Lasenby, M. Lattanzi, C. R. Lawrence, J. P. Leahy, R. Leonardi, J. Lesgourgues, F. Levrier, A. Lewis, M. Liguori, P. B. Lilje, M. Linden-Vørnle, M. López-Caniego, P. M. Lubin, J. F. Macías-Pérez, G. Maggio, D. Maino, N. Mandolesi, A. Mangilli, A. Marchini, M. Maris, P. G. Martin, M. Martinelli, E. Martínez-González, S. Masi, S. Matarrese, P. McGehee, P. R. Meinhold, A. Melchiorri, J.-B. Melin, L. Mendes, A. Mennella, M. Migliaccio, M. Millea, S. Mitra, M.-A. Miville-Deschênes, A. Moneti, L. Montier, G. Morgante, D. Mortlock, A. Moss, D. Munshi, J. A. Murphy, P. Naselsky, F. Nati, P. Natoli, C. B. Netterfield, H. U. Nørgaard-Nielsen, F. Noviello, D. Novikov, I. Novikov, C. A. Oxborrow, F. Paci, L. Pagano, F. Pajot, R. Paladini, D. Paoletti, B. Partridge, F. Pasian, G. Patanchon, T. J. Pearson, O. Perdereau, L. Perotto, F. Perrotta, V. Pettorino, F. Piacentini, M. Piat, E. Pierpaoli, D. Pietrobon, S. Plaszczynski, E. Pointecouteau, G. Polenta, L. Popa, G. W. Pratt, G. Prézeau, S. Prunet, J.-L. Puget, J. P. Rachen, W. T. Reach, R. Rebolo, M. Reinecke, M. Remazeilles, C. Renault, A. Renzi, I. Ristorcelli, G. Rocha, C. Rosset, M. Rossetti, G. Roudier, B. Rouillé d'Orfeuil, M. Rowan-Robinson, J. A. Rubiño-Martín, B. Rusholme, N. Said, V. Salvatelli, L. Salvati, M. Sandri, D. Santos, M. Savelainen, G. Savini, D. Scott, M. D. Seiffert, P. Serra, E. P. S. Shellard, L. D. Spencer, M. Spinelli, V. Stolyarov, R. Stompor, R. Sudiwala, R. Sunyaev, D. Sutton, A.-S. Suur-Uski, J.F. Sygnet, J. A. Tauber, L. Terenzi, L. Toffolatti, M. Tomasi, M. Tristram, T. Trombetti, M. Tucci, J. Tuovinen, M. Türler, G. Umana, L. Valenziano, J. Valiviita, F. Van Tent, P. Vielva, F. Villa, L. A. Wade, B. D. Wandelt, I. K. Wehus, M. White, S. D. M. White, A. Wilkinson, D. Yvon, A. Zacchei, A. Zonca; Planck Collaboration, Planck 2015 results. XIII. Cosmological parameters. Astron. Astrophys. 594, A13 (2016). doi:10.1051/0004-6361/201525830

33. K. W. Bannister, A. T. Deller, C. Phillips, J.-P. Macquart, J. X. Prochaska, N. Tejos, S. D. Ryder, E. M. Sadler, R. M. Shannon, S. Simha, C. K. Day, M. McQuinn, F. O. North-Hickey, S. Bhandari, W. R. Arcus, V. N. Bennert, J. Burchett, M. Bouwhuis, R. Dodson, R. D. Ekers, W. Farah, C. Flynn, C. W. James, M. Kerr, E. Lenc, E. K. Mahony, J. O'Meara, S. Osłowski, H. Qiu, T. Treu, V. U, T. J. Bateman, D. C.-J. Bock, R. J. Bolton, A. Brown, J. D. Bunton, A. P. Chippendale, F. R. Cooray, T. Cornwell, N. Gupta, D. B. Hayman, M. Kesteven, B. S. Koribalski, A. MacLeod, N. M. McClureGriffiths, S. Neuhold, R. P. Norris, M. A. Pilawa, R.-Y. Qiao, J. Reynolds, D. N. Roxby, T. W. Shimwell, M. A. Voronkov, C. D. Wilson, A single fast radio burst localized to a massive galaxy at cosmological distance. Science 365, 565-570 (2019). doi:10.1126/science.aaw5903 Medline

34. A. T. Deller, W. F. Brisken, C. J. Phillips, J. Morgan, W. Alef, R. Cappallo, E. Middelberg, J. Romney, H. Rottmann, S. J. Tingay, R. Wayth, DiFX-2: A More Flexible, Efficient, Robust, and Powerful Software Correlator. Publ. Astron. Soc. Pac. 123, 275-287 (2011). doi:10.1086/658907

35. E. W. Greisen, Information Handling in Astronomy - Historical Vistas, A. Heck, Ed. (2003), vol. 285 of Astrophysics and Space Science Library, p. 109.

36. M. Kettenis, H. J. van Langevelde, C. Reynolds, B. Cotton, Astronomical Data Analysis Software and Systems XV, C. Gabriel, C. Arviset, D. Ponz, S. Enrique, Eds. (2006), vol. 351 of Astronomical Society of the Pacific Conference Series, p. 497.

37. J. P. McMullin, B. Waters, D. Schiebel, W. Young, K. Golap, Astronomical Data Analysis Software and Systems XVI, R. A. Shaw, F. Hill, D. J. Bell, Eds. (2007), vol. 376 of Astronomical Society of the Pacific Conference Series, p. 127.

38. R. J. Sault, P. J. Teuben, M. C. H. Wright, Astronomical Data Analysis Software and Systems IV, R.Shaw, H. Payne, J. J. E. Hayes, Eds. (1995), vol. 77 of Astronomical Society of the Pacific Conference Series, p.433.

39. J. E. Everett, J. M. Weisberg, Emission Beam Geometry of Selected Pulsars Derived from Average Pulse Polarization Data. Astrophys. J. 553, 341-357 (2001). doi:10.1086/320652

40. F. Feroz, M. P. Hobson, M. Bridges, MultiNest: An efficient and robust Bayesian inference tool for cosmology and particle physics. Mon. Not. R. Astron. Soc. 398 , 1601-1614 (2009). doi:10.1111/i.1365-2966.2009.14548.x

41. E. L. Wright, P. R. M. Eisenhardt, A. K. Mainzer, M. E. Ressler, R. M. Cutri, T. Jarrett, J. D. Kirkpatrick, D. Padgett, R. S. McMillan, M. Skrutskie, S. A. Stanford, M. Cohen, R. G. Walker, J. C. Mather, D. Leisawitz, T. N. Gautier, I. McLean, D. Benford, C. J. Lonsdale, A. Blain, B. Mendez, W. R. Irace, V. Duval, F. Liu, D. Royer, I. Heinrichsen, J. Howard, M. Shannon, M. Kendall, A. L. Walsh, M. Larsen, J. G. Cardon, S. Schick, M. Schwalm, M. Abid, B. Fabinsky, L. Naes, C.-W. Tsai, The Wide-field Infrared Survey Explorer (WISE): Mission Description and Initial On-orbit Performance. Astron. J. 140, 1868-1881 (2010). doi:10.1088/0004-6256/140/6/1868
42. I. Appenzeller et al., Successful commissioning of FORS1 - the first optical instrument on the VLT. The Messenger 94, 1 (1998).

43. G. Berriman, J. Good, The Application of the Montage Image Mosaic Engine to the Visualization of Astronomical Images. Publ. Astron. Soc. Pac. 129, 058006 (2017). doi:10.1088/1538-3873/aa5456

44. D. Lang, D. W. Hogg, K. Mierle, M. Blanton, S. Roweis, Astrometry.net: Blind astrometric calibration of arbitrary astronomical images. Astron. J. 139, 17821800 (2010). doi:10.1088/0004-6256/139/5/1782

45. E. Bertin, S. Arnouts, SExtractor: Software for source extraction. Astron. Astrophys. Suppl. Ser. 117, 393-404 (1996). doi:10.1051/aas:1996164

46. Pypelt, The Python Spectroscopic Data Reduction Pipeline; https://pypeit.readthedocs.io.

47. E. Platts et al., arXiv:1810.05836 (2018)

48. S. Noll, D. Burgarella, E. Giovannoli, V. Buat, D. Marcillac, J. C. Muñoz-Mateos, Analysis of galaxy spectral energy distributions from far-UV to far-IR with CIGALE: Studying a SINGS test sample. Astron. Astrophys. 507, 1793-1813 (2009). doi:10.1051/0004-6361/200912497

49. G. Bruzual, S. Charlot, Stellar population synthesis at the resolution of 2003. Mon. Not. R. Astron. Soc. 344, 1000-1028 (2003). doi:10.1046/j.13658711.2003.06897x

50. D. Calzetti, L. Armus, R. C. Bohlin, A. L. Kinney, J. Koornneef, T. Storchi-Bergmann, The Dust Content and Opacity of Actively Star-forming Galaxies. Astrophys. J. 533, 682-695 (2000). doi:10.1086/308692

51. D. A. Dale, G. Helou, G. E. Magdis, L. Armus, T. Díaz-Santos, Y. Shi, A two-parameter model for the infrared/submillimeter/radio spectral energy distributions of galaxies and active galactic nuclei. Astrophys. J. 784, 83 (2014). doi:10.1088/0004-637X/784/1/83

52. M. Cappellari, Improving the full spectrum fitting method: Accurate convolution with Gauss-Hermite functions. Mon. Not. R. Astron. Soc. 466, 798-811 (2017). doi:10.1093/mnras/stw3020

53. L. J. Kewley, M. A. Dopita, R. S. Sutherland, C. A. Heisler, J. Trevena, Theoretical Modeling of Starburst Galaxies. Astrophys. J. 556, 121-140 (2001). doi:10.1086/321545

54. G. Kauffmann, T. M. Heckman, C. Tremonti, J. Brinchmann, S. Charlot, S. D. M. White, S. E. Ridgway, J. Brinkmann, M. Fukugita, P. B. Hall, Ž. Ivezić, G. T. Richards, D. P. Schneider, The host galaxies of active galactic nuclei. Mon. Not. R. Astron. Soc. 346, 1055-1077 (2003). doi:10.1111/j.1365-2966.2003.07154.x

55. R. Cid Fernandes, G. Stasińska, M. S. Schlickmann, A. Mateus, N. Vale Asari, W. Schoenell, L. Sodré Jr., Alternative diagnostic diagrams and the 'forgotten' population of weak line galaxies in the SDSS. Mon. Not. R. Astron. Soc. 403, 10361053 (2010). doi:10.1111/j.1365-2966.2009.16185.x

56. S. P. Tendulkar, C. G. Bassa, J. M. Cordes, G. C. Bower, C. J. Law, S. Chatterjee, E. A. K. Adams, S. Bogdanov, S. Burke-Spolaor, B. J. Butler, P. Demorest, J. W. T. Hessels, V. M. Kaspi, T. J. W. Lazio, N. Maddox, B. Marcote, M. A. McLaughlin, Z. Paragi, S. M. Ransom, P. Scholz, A. Seymour, L. G. Spitler, H. J. van Langevelde, R. S. Wharton, The Host Galaxy and Redshift of the Repeating Fast Radio Burst FRB 121102. Astrophys. J. 834, L7 (2017). doi:10.3847/2041-8213/834/2/L7

57. V. Ravi, M. Catha, L. D’Addario, S. G. Djorgovski, G. Hallinan, R. Hobbs, J. Kocz, S. R. Kulkarni, J. Shi, H. K. Vedantham, S. Weinreb, D. P. Woody, A fast radio burst localized to a massive galaxy. Nature 572, 352-354 (2019). doi:10.1038/s41586019-1389-7 Medline

58. J. Moustakas, A. L. Coil, J. Aird, M. R. Blanton, R. J. Cool, D. J. Eisenstein, A. J. Mendez, K. C. Wong, G. Zhu, S. Arnouts, PRIMUS: Constraints on star formation quenching and galaxy merging, and the evolution of the stellar mass function from $z=0$-1. Astrophys. J. 767, 50 (2013). doi:10.1088/0004-637X/767/1/50

59. M. Nicholl, P. K. G. Williams, E. Berger, V. A. Villar, K. D. Alexander, T. Eftekhari, B. D. Metzger, Empirical Constraints on the Origin of Fast Radio Bursts: Volumetric Rates and Host Galaxy Demographics as a Test of Millisecond Magnetar Connection. Astrophys. J. 843, 84 (2017). doi:10.3847/1538-4357/aa794d

60. C. Y. Peng, L. C. Ho, C. D. Impey, H.-W. Rix, Detailed decomposition of galaxy images. II. Beyond axisymmetric models. Astron. J. 139, 2097-2129 (2010). doi:10.1088/0004-6256/139/6/2097

61. L. Bradley et al., 10.5281/zenodo.2533376.

62. B. P. Moster, R. S. Somerville, C. Maulbetsch, F. C. van den Bosch, A. V. Macciò, T. Naab, L. Oser, Constraints on the relationship between stellar mass and halo mass 
at low and high redshift. Astrophys. J. 710, 903-923 (2010). doi:10.1088/0004$637 X / 710 / 2 / 903$

63. J. DeRose, R. H. Wechsler, J. L. Tinker, M. R. Becker, Y.-Y. Mao, T. McClintock, S. McLaughlin, E. Rozo, Z. Zhai, The Aemulus Project. I. Numerical Simulations for Precision Cosmology. Astrophys. J. 875, 69 (2019). doi:10.3847/15384357/ab1085

64. J. M. Cordes, T. J. W. Lazio, arXiv:astro-ph/0207156 (2002).

65. J. Navarro, C. Frenk, S. White, A Universal Density Profile from Hierarchical Clustering. Astrophys. J. 490, 493-508 (1997). doi:10.1086/304888

66. D. Fielding, E. Quataert, M. McCourt, T. A. Thompson, The impact of star formation feedback on the circumgalactic medium. Mon. Not. R. Astron. Soc. 466, 38103826 (2017). doi:10.1093/mnras/stw3326

67. M. Su, T. R. Slatyer, D. P. Finkbeiner, Giant gamma-ray bubbles from Fermi-LAT: Active galactic nucleus activity or bipolar galactic wind? Astrophys. J. 724, 10441082 (2010). doi:10.1088/0004-637X/724/2/1044

68. R. Bordoloi, A. J. Fox, F. J. Lockman, B. P. Wakker, E. B. Jenkins, B. D. Savage, S. Hernandez, J. Tumlinson, J. Bland-Hawthorn, T.-S. Kim, Mapping the nuclear outflow of the Milky Way: Studying the kinematics and spatial extent of the northern Fermi Bubble. Astrophys. J. 834, 191 (2017). doi:10.3847/1538$4357 / 834 / 2 / 191$

69. M. T. Karim, A. J. Fox, E. B. Jenkins, R. Bordoloi, B. P. Wakker, B. D. Savage, F. J. Lockman, S. M. Crawford, R. A. Jorgenson, J. Bland-Hawthorn, Probing the Southern Fermi Bubble in Ultraviolet Absorption Using Distant AGNs. Astrophys. J. 860, 98 (2018). doi:10.3847/1538-4357/aac167

70. E. Carretti, R. M. Crocker, L. Staveley-Smith, M. Haverkorn, C. Purcell, B. M. Gaensler, G. Bernardi, M. J. Kesteven, S. Poppi, Giant magnetized outflows from the centre of the Milky Way. Nature 493, 66-69 (2013). doi:10.1038/nature11734 Medline

71. N. Oppermann, H. Junklewitz, G. Robbers, M. R. Bell, T. A. Enßlin, A. Bonafede, R. Braun, J. C. Brown, T. E. Clarke, I. J. Feain, B. M. Gaensler, A. Hammond, L. HarveySmith, G. Heald, M. Johnston-Hollitt, U. Klein, P. P. Kronberg, S. A. Mao, N. M. McClure-Griffiths, S. P. O'Sullivan, L. Pratley, T. Robishaw, S. Roy, D. H. F. M. Schnitzeler, C. Sotomayor-Beltran, J. Stevens, J. M. Stil, C. Sunstrum, A. Tanna, A. R. Taylor, C. L. Van Eck, An improved map of the Galactic Faraday sky. Astron. Astrophys. 542, A93 (2012). doi:10.1051/0004-6361/201118526

72. A. H. Maller, J. S. Bullock, Multiphase galaxy formation: High-velocity clouds and the missing baryon problem. Mon. Not. R. Astron. Soc. 355, 694-712 (2004). doi:10.1111/i.1365-2966.2004.08349.x

73. A. Woodfinden, R. N. Henriksen, J. Irwin, S. C. Mora-Partiarroyo, Evolving galactic dynamos and fits to the reversing rotation measures in the halo of NGC 4631. Mon. Not. R. Astron. Soc. 487, 1498-1516 (2019). doi:10.1093/mnras/stz1366

74. W. A. Coles, R. G. Frehlich, B. J. Rickett, J. L. Codona, Refractive scintillation in the interstellar medium. Astrophys. J. 315, 666 (1987). doi:10.1086/165168

75. J. W. Armstrong, B. J. Rickett, S. R. Spangler, Electron density power spectrum in the local interstellar medium. Astrophys. J. 443, 209 (1995). doi:10.1086/175515

76. L. C. Lee, J. R. Jokipii, Strong scintillations in astrophysics. II - A theory of temporal broadening of pulses. Astrophys. J. 201, 532 (1975). doi:10.1086/153916

77. M. McQuinn, J. K. Werk, Implications of the Large 0 vi Columns around Lowredshift L * Galaxies. Astrophys. J. 852, 33 (2018). doi:10.3847/15384357/aa9d3f

78. Y. Lithwick, P. Goldreich, Compressible Magnetohydrodynamic Turbulence in Interstellar Plasmas. Astrophys. J. 562, 279-296 (2001). doi:10.1086/323470

79. P. Goldreich, S. Sridhar, Toward a theory of interstellar turbulence. 2: Strong alfvenic turbulence. Astrophys. J. 438, 763 (1995). doi:10.1086/175121

80. M. McCourt, P. Sharma, E. Quataert, I. J. Parrish, Thermal instability in gravitationally stratified plasmas: Implications for multiphase structure in clusters and galaxy haloes. Mon. Not. R. Astron. Soc. 419, 3319-3337 (2012). doi:10.1111/j.1365-2966.2011.19972.x

81. H. K. Vedantham, E. S. Phinney, Radio wave scattering by circumgalactic cool gas clumps. Mon. Not. R. Astron. Soc. 483, 971-984 (2019). doi:10.1093/mnras/sty2948

82. B. T. Draine, Physics of the Interstellar and Intergalactic Medium (2011).

83. J. S. Morgan, M. K. Argo, C. M. Trott, J.-P. Macquart, A. Deller, E. Middelberg, J. Miller-Jones, S. J. Tingay, Wide-field VLBI observations of M31: A unique probe of the ionized interstellar medium of a nearby galaxy. Astrophys. J. 768, 12 (2013).
doi:10.1088/0004-637X/768/1/12

84. S. R. Spangler, C. R. Gwinn, Evidence for an inner scale to the density turbulence in the interstellar medium. Astrophys. J. 353, L29 (1990). doi:10.1086/185700

85. W. Farah, C. Flynn, M. Bailes, A. Jameson, K. W. Bannister, E. D. Barr, T. Bateman, S. Bhandari, M. Caleb, D. Campbell-Wilson, S.-W. Chang, A. Deller, A. J. Green, R. Hunstead, F. Jankowski, E. Keane, J.-P. Macquart, A. Möller, C. A. Onken, S. Osłowski, A. Parthasarathy, K. Plant, V. Ravi, R. M. Shannon, B. E. Tucker, V. Venkatraman Krishnan, C. Wolf, FRB microstructure revealed by the real-time detection of FRB170827. Mon. Not. R. Astron. Soc. 478, 1209-1217 (2018). doi:10.1093/mnras/sty1122

86. M. Ackermann, A. Albert, W. B. Atwood, L. Baldini, J. Ballet, G. Barbiellini, D. Bastieri, R. Bellazzini, E. Bissaldi, R. D. Blandford, E. D. Bloom, E. Bottacini, T. J. Brandt, J. Bregeon, P. Bruel, R. Buehler, S. Buson, G. A. Caliandro, R. A. Cameron, M. Caragiulo, P. A. Caraveo, E. Cavazzuti, C. Cecchi, E. Charles, A. Chekhtman, J. Chiang, G. Chiaro, S. Ciprini, R. Claus, J. Cohen-Tanugi, J. Conrad, S. Cutini, F. D'Ammando, A. de Angelis, F. de Palma, C. D. Dermer, S. W. Digel, L. D. Venere, E. do Couto e Silva, P. S. Drell, C. Favuzzi, E. C. Ferrara, W. B. Focke, A. Franckowiak, Y. Fukazawa, S. Funk, P. Fusco, F. Gargano, D. Gasparrini, S. Germani, N. Giglietto, F. Giordano, M. Giroletti, G. Godfrey, G. A. Gomez-Vargas, I. A. Grenier, S. Guiriec, D. Hadasch, A. K. Harding, E. Hays, J. W. Hewitt, X. Hou, T. Jogler, G. Jóhannesson, A. S. Johnson, W. N. Johnson, T. Kamae, J. Kataoka, J. Knödlseder, D. Kocevski, M. Kuss, S. Larsson, L. Latronico, F. Longo, F. Loparco, M. N. Lovellette, P. Lubrano, D. Malyshev, A. Manfreda, F. Massaro, M. Mayer, M. N. Mazziotta, J. E. McEnery, P. F. Michelson, W. Mitthumsiri, T. Mizuno, M. E. Monzani, A. Morselli, I. V. Moskalenko, S. Murgia, R. Nemmen, E. Nuss, T. Ohsugi, N. Omodei, M. Orienti, E. Orlando, J. F. Ormes, D. Paneque, J. H. Panetta, J. S. Perkins, M. Pesce-Rollins, V. Petrosian, F. Piron, G. Pivato, S. Rainò, R. Rando, M. Razzano, S. Razzaque, A. Reimer, O. Reimer, M. Sánchez-Conde, M. Schaal, A. Schulz, C. Sgrò, E. J. Siskind, G. Spandre, P. Spinelli, Ł. Stawarz, A. W. Strong, D. J. Suson, M. Tahara, H. Takahashi, J. B. Thayer, L. Tibaldo, M. Tinivella, D. F. Torres, G. Tosti, E. Troja, Y. Uchiyama, G. Vianello, M. Werner, B. L. Winer, K. S. Wood, M. Wood, G. Zaharijas, The spectrum and morphology of the Fermi bubbles. Astrophys. J. 793, 64 (2014). doi:10.1088/0004-637X/793/1/64

\section{ACKNOWLEDGMENTS}

We are grateful to Australia Telescope National Facility (ATNF) operations staff and the Murchison Radio-astronomy Observatory staff for supporting our ASKAP operations, and the ATNF Director and Steering Committee for dedicating time for these observations. Work at the Naval Research Laboratory is supported by NASA. The Australian Square Kilometre Array Pathfinder, Australia Telescope Compact Array, and Parkes Radio Telescope are part of the Australia Telescope National Facility which is managed by CSIRO. Operation of ASKAP is funded by the Australian Government with support from the National Collaborative Research Infrastructure Strategy. ASKAP uses the resources of the Pawsey Supercomputing Centre. Establishment of ASKAP, the Murchison Radioastronomy Observatory and the Pawsey Supercomputing Centre are initiatives of the Australian Government, with support from the Government of Western Australia and the Science and Industry Endowment Fund. We acknowledge the Wajarri Yamatji as the traditional owners of the Murchison Radio-astronomy Observatory site. Funding: J.X.P. and S.S. are supported by the National Science Foundation grant AST-1911140. K.W.B., J.P.M, and R.M.S. acknowledge Australian Research Council (ARC) grant DP180100857. A.T.D. is the recipient of an ARC Future Fellowship (FT150100415). R.M.S. acknowledge support through ARC grant FL150100148 and through ARC grant CE170100004. N.T. acknowledges support from PUCV research funding 039.333/2018. Author contributions: J.X.P., J.P.M., M.M., S.S., R.M.S, C.F., and C.K.D drafted the manuscript. J.X.P., N.T., S.R., L.M, S.S., and E.M. obtained, reduced and interpreted optical observations. R.B. performed the Fermi Bubble analysis. K.W.B. built the search and voltage capture software. A.D., C.P., C.K.D. H.C.,H.Q. and S.B., designed, built and conducted the correlation, calibration and imaging software to localize FRB 181112. R.M.S. led the ASKAP observing, and interpreted radio band polarization data. E.M. and S.B. obtained, reduced, and interpreted ATCA data. J.B. designed the ASKAP digital systems. Competing interests: The authors declare no competing interests. Data and materials availability: Based on observations collected at the European Southern Observatory, available from 
http://archive.eso.org/ under program ID 0102.A-0450(A) (PI: Macquart); before the ESO proprietary period expires they can be obtained at https://drive.google.com/drive/folders/150rdfZqJbNAnj-

mGdOHX9t8nSBLcf9sZ. Observations from the Australia Telescope Compact Array are available at (30). Further datasets used in this paper are available from the gSTAR Data Management and Collaboration Platform (gDMCP) at https://data-portal.hpc.swin.edu.au/dataset/askap-visibilities-and-images-forfrb181112: the nine ASKAP visibility datasets used to calibrate and determine the localization of FRB 181112, radio images of the FRB and surrounding field, and the ATCA images used for astrometric alignment. Reduced data and scripts are available at (31). Data reduction scripts and code written by the co-authors for this project are available from the craft git repository

https://bitbucket.csiro.au/scm/craf/craft.git, the psrvlbireduce repository

https://github.com/dingswin/psrvlbireduce, the FRB repository

https://github.com/FRBs/FRB, and the Pypelt repository

https://github.com/pypeit/pypeit.

\section{SUPPLEMENTARY MATERIALS}

science.sciencemag.org/cgi/content/full/science.aay0073/DC1 Materials and Methods

Supplementary Text

Figs. S1 to S12

Tables S1 to S7

References (32-86)

20 May 2019; accepted 17 September 2019

Published online 26 September 2019

10.1126/science.aay0073
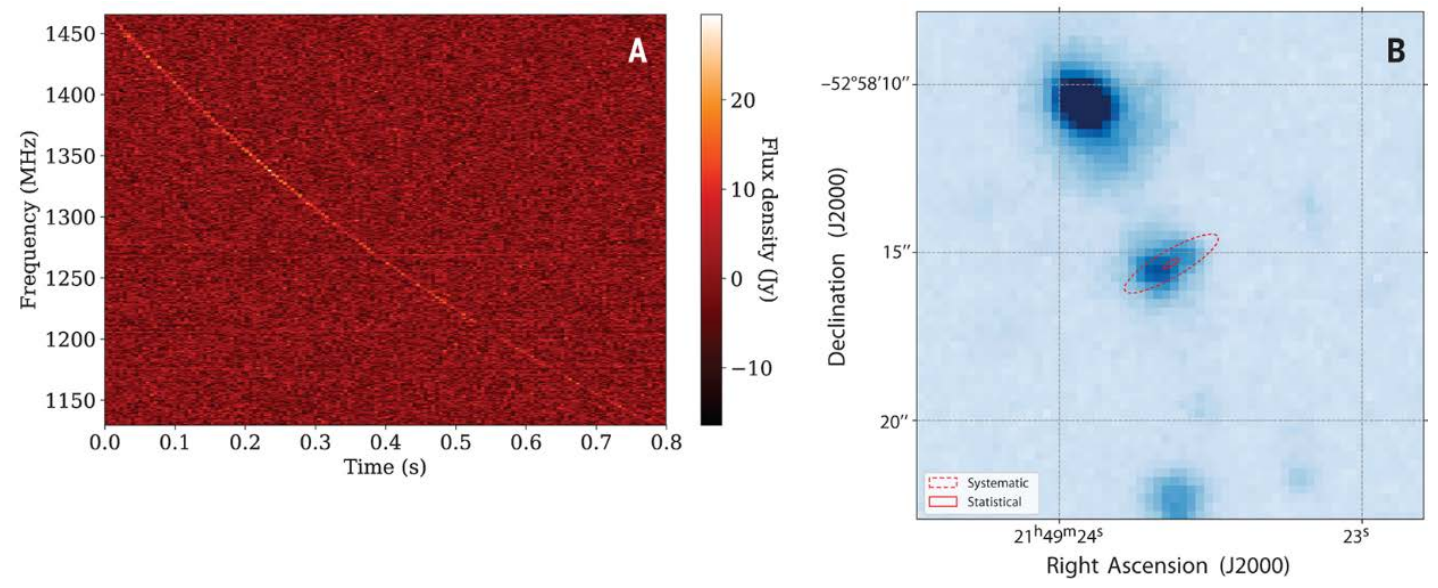

Fig. 1. Dynamic spectrum of FRB 181112 and optical imaging of its host and a coincident foreground galaxy. (A) Dynamic spectrum of FRB 181112 recorded by ASKAP. The dispersion measure DM FRB $=589.27 \mathrm{pc} \mathrm{cm}^{-3}$; (B) $g$-band FORS2 image centered on FRB 181112 whose position is depicted by the red ellipses with solid/dashed lines indicating the statistical/systematic uncertainty. We estimate an additional systematic uncertainty of $\approx 0.5^{\prime \prime}$ in the astrometric solution of the FORS2 image. The host is well-localized to a faint galaxy cataloged as DES J214923.66-525815.28, and one identifies a brighter galaxy located $\approx 0.5^{\prime \prime}$ away at a $P A \approx 13^{\circ}$ (cataloged as DES J214923.89-525810.43, referred to as FG-181112). The sightline to FRB 181112 passes through the halo of this foreground galaxy at an impact parameter $R_{\perp}=29 \mathrm{kpc}$. 

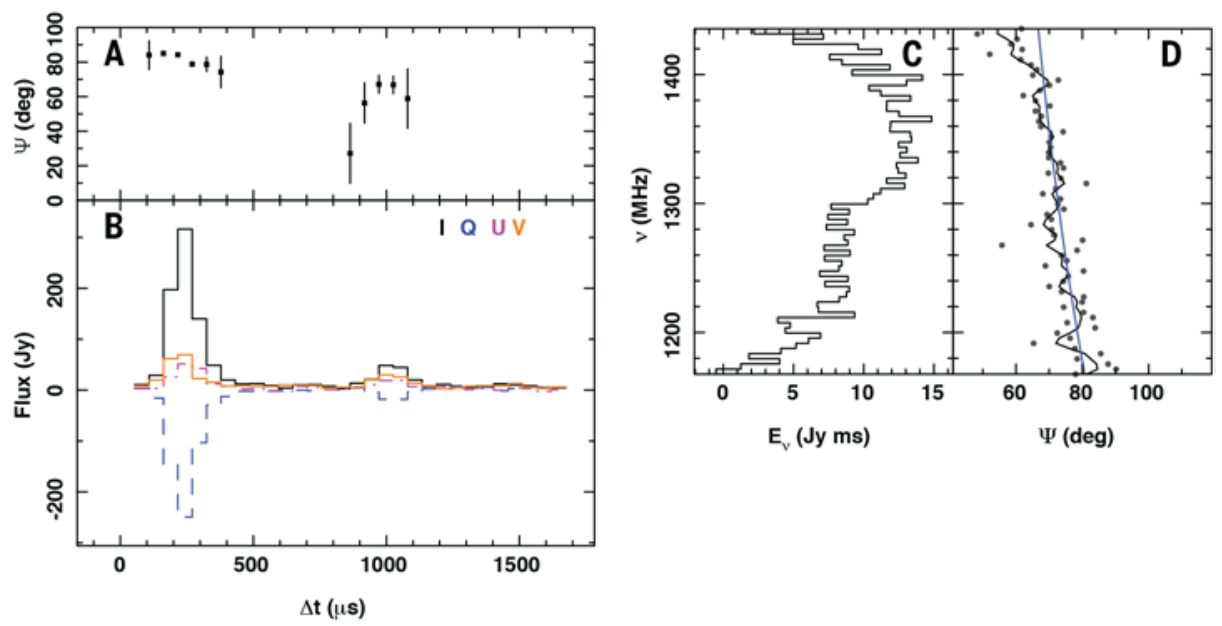

Fig. 2. Spectropolarimeric properties of FRB 181112. (A) Relative linear polarization position angle $\Psi$ of the burst averaged in frequency. (B) Polarimetric pulse profile of burst in four Stokes components (I - thick solid line; Q dashed line; U - dashed-dotted line, V thin solid line). The two components, separated by approximately $800 \mu$ s show different position angles. (C) Spectrum of $E_{v}$ burst averaged over both pulses. (D) Position angle $\Psi$ of the burst plotted as a function of frequency, with the black points showing measurements in individual frequency channels, and line these measurements smoothed using a Gaussian Kernel with standard deviation of $4 \mathrm{MHz}$. The variation of the position angle with frequency is the result of Faraday rotation. The blue line shows a maximum-likelihood model for polarization, using the inferred rotation measure $\mathrm{RM}=10.9 \mathrm{rad} \mathrm{m}^{-2}$.
Fig. 3. Constraints on the coherent magnetic field parallel to the line of sight $B_{\|}$and electron density $n_{e}$ in the halo of FG-181112. The hatched regions show the parameter space in $B_{\|}, n_{e}$ (cool gas) ruled out for the halo of FG-181112 from the measured RM and $\tau_{\text {scatt }}$ of FRB 181112. These constraints are largely independent of the properties of the foreground galaxy. We compare these results with previous inferences for the density of cool halo gas (colored regions) based on ionization modeling and $L y \alpha$ fluorescence. We also illustrate previous estimations for the magnetic field strength in halo gas (yellow curve; (22)) which conflict with our results.

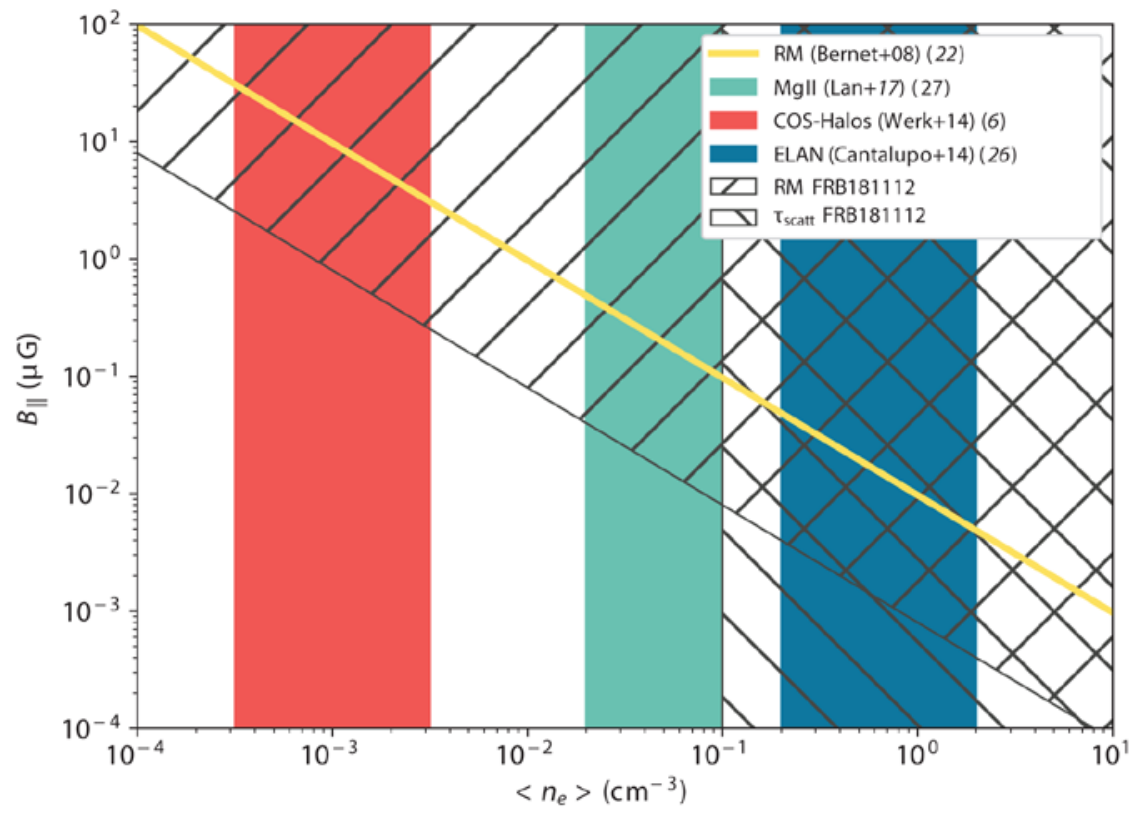

www.sciencemag.org (Page numbers not final at time of first release) 
Table 1. Properties of FRB 181112, its host, and the foreground galaxy FG-181112. The two uncertainties in Right Ascension and Declination are statistical and systematic, projected onto the coordinate axes. These uncertainties are best described as ellipses with position angle $120 \mathrm{deg}$ East of North and major/minor axes of $\left(a_{\text {statistical }}=0.55^{\prime \prime},, b_{\text {statistical }}\right.$ $\left.=0.15^{\prime \prime}\right)$ and $\left(a_{\text {systematic }}=3.2^{\prime \prime}, b_{\text {systematic }}=0.8^{\prime \prime}\right)$. The coherent magnetic field, density and filling factor estimates assume a characteristic path length through the halo of $\Delta L=50 \mathrm{kpc}$. The density and filling factor estimates assume a driving scale with root mean density fluctuations of one at $L_{0}=1 \mathrm{kpc}$, with the bound scaling as $\propto L_{0}^{1 / 3}$, as well as a Kolmogorov spectrum of turbulence to separations below $r_{\text {diff. }}$. The filling factor estimate further assumes cool $T_{\text {cool }}=10^{4} \mathrm{~K}$ gas is in pressure equilibrium with hot gas with density $\left\langle n_{e}\right\rangle=10^{-3} \mathrm{~cm}^{-3}$ and temperature $T_{\text {hot }}=2 \times 10^{6} \mathrm{~K}$ hot gas, with the bound scaling as $\propto\left(\left\langle n_{e}\right\rangle T_{\text {hot }} / T_{\text {cool }}\right)^{-2}$. See text and (12) for details.

\begin{tabular}{ll}
\hline FRB & \\
Right Ascension (J2000) & $327.34846 \pm 0.00007 \pm 0.0006 \mathrm{deg}$ \\
Declination (J2000) & $-52.97093 \pm 0.00004 \pm 0.0002 \mathrm{deg}$ \\
Dispersion measure (DM FRB) & $589.27 \pm 0.03 \mathrm{pc} \mathrm{cm}^{-3}$ \\
Rotation measure (RM) & $10.9 \pm 0.9 \mathrm{rad} \mathrm{m}^{-2}$ \\
Pulse width & $<40 \mu \mathrm{S}$ \\
Host Galaxy & $0.47550 \pm 0.00015$ \\
Redshift & $2.6 \pm 1.1 \times 10^{9} M_{\odot}$ \\
Stellar Mass & $0.6 M_{\odot} \mathrm{yr}^{-1}$ \\
Star formation rate & \\
Foreground galaxy FG-181112 & $0.36738 \pm 0.00007$ \\
Redshift & $29 \pm 3 \mathrm{kpc}^{-}$ \\
Impact parameter to the FRB sightline $\left(R_{\perp}\right)$ & $4.9 \pm 3.2 \times 10^{10} M_{\odot}$ \\
Stellar Mass & $<0.3 M_{\odot} \mathrm{yr}^{-1}$ \\
Star formation rate & $B_{\|}<0.5 \mu \mathrm{G}\left(\mathrm{n}_{e} / 10^{-3} \mathrm{~cm}^{-3}\right)$ \\
Coherent magnetic field parallel to the line of sight & $n_{e}<2 \times 10^{-3} \mathrm{~cm}^{-3}$ \\
Density constraint for hot, diffuse gas $\left(f_{V} \sim 1\right)$ & $f_{V}<10^{-4}$ \\
\hline Filling factor constraint for cool, clumpy gas & \\
\hline
\end{tabular}

